\title{
Test for the Radio Detection of the Extensive Air Shower using the Electron Beam in Telescope Array
}

\section{Daisuke Ikeda $^{* a}$, for the Telescope Array Collaboration ${ }^{\dagger}$}

${ }^{a}$ Institute for the Cosmic Ray Research, University of Tokyo, Chiba, Japan

E-mail: ikeda@icrr.u-tokyo.ac.jp

For the future huge ultra-high energy cosmic ray observatory, we are carrying out an R\&D project to search for radar echoes from cosmic ray induced extensive air showers. First the experiment with the electron beam which is generated by the Electron Light Source (ELS) in the TA site was performed. We did not find any radar echo signals but found the radio emission signals proportional to the beam current. Second we developed new experimental setup to see the radar echoes and radio emissions from the air shower in the TA site. The construction was finished on July 2014 and steady operation was started from October 2014. The design of the experiments and results of the observed signals are presented.

The 34th International Cosmic Ray Conference,

30 July- 6 August, 2015

The Hague, The Netherlands

\footnotetext{
* Speaker.

${ }^{\dagger}$ For full author list and acknowledgments see http://www.telescopearray.org/images/papers/ICRC2015authorlist.pdf
} 


\section{Introduction}

One of the directions of the future experiments of the ultra-high energy cosmic rays (UHECRs) is to collect more statistics. The current large observatory, the Telescope Array (TA) [1] and Auger Observatory [2], are driven by two observation techniques. One is the surface detectors (SDs) detecting secondary particles of the air shower on the ground, and the other is the fluorescence detectors (FDs) observing the fluorescence light emitted along the air shower in the atmosphere. A new technique, radar echo detection [3], has the capability of using both of the strengths of those detectors: operating 100\% duty cycle like SD and observing the air shower longitudinal development like FD. In addition, one receiver will cover the large observation area so that the maintenance is slightly easier compared with the SD array.

To confirm and develop this technique for the future huge UHECR observatory, we carried out the R\&D experiment by using the electron beam from the Electron Light Source (ELS) [4] in the TA site. By this experiment, we did not observe any clear signals of the radar echo but observed the radio emission from the electron beam. One of the possibilities to describe the observed signal is an electric field generated by the sudden appearing of the electron beam from the ELS container. It is likely a phenomenon opposite to be a sudden vanishing of the air shower's secondary particles at the ground (sudden death) [5].

Under the situation mentioned the above, we developed a new experiment in the TA site, observing the radio signal from the air showers with the TA detectors. Because the other experiments to observe the radar echoes, TARA [6], is running in the TA site and transmitting the radio, those receivers have a capability to observe the radar echoes from the air showers. In addition, because those are installed at the center of the SD arrays, the sudden death signal is also expected to be observed with the near SDs.

The design of these experiments and result of the electron beam test in the TA site are presented.

\section{Design of the electron beam test}

The basic idea of this experiment is to search for radar echo signals from the electron beam induced by ELS in the TA site. The positions of the transmitter, receiver and ELS are shown in Figure 1.

ELS was installed in front of the FD for the absolute calibration. The ELS shoots the electrons of $40 \mathrm{MeV}$ energy, $1 \mu \mathrm{s}$ beam duration and up to $160 \mathrm{pC}$ to the atmosphere vertically. In addition, to increase the sensitivity in the limitation of the total output charge, we used a short pulse beam of $20 \mathrm{~ns}$ width as well as a long pulse of $1 \mu$ s width.

For the radio echo search, the radio transmitter and receiver were used. We installed two 54.1 MHz transmitters in the TA site. One was installed $21 \mathrm{~km}$ distant from ELS (TX1), and the other was $140 \mathrm{~m}$ distant from the ELS (TX2). Both of transmitters emit the continuous radio wave with up to $2 \mathrm{~kW}$ (TX1) and up to $100 \mathrm{~W}$ (TX2). The polarization can be changed by rotating the antenna.

To detect the scattered radio wave by the electro-magnetic shower induced by the ELS beam, two receivers are installed $140 \mathrm{~m}$ from the ELS. The developed receiver consists of log-periodic antenna, 30dB pre-amplifier and a digitizer. We used a software-defined radio (SDR) system with 


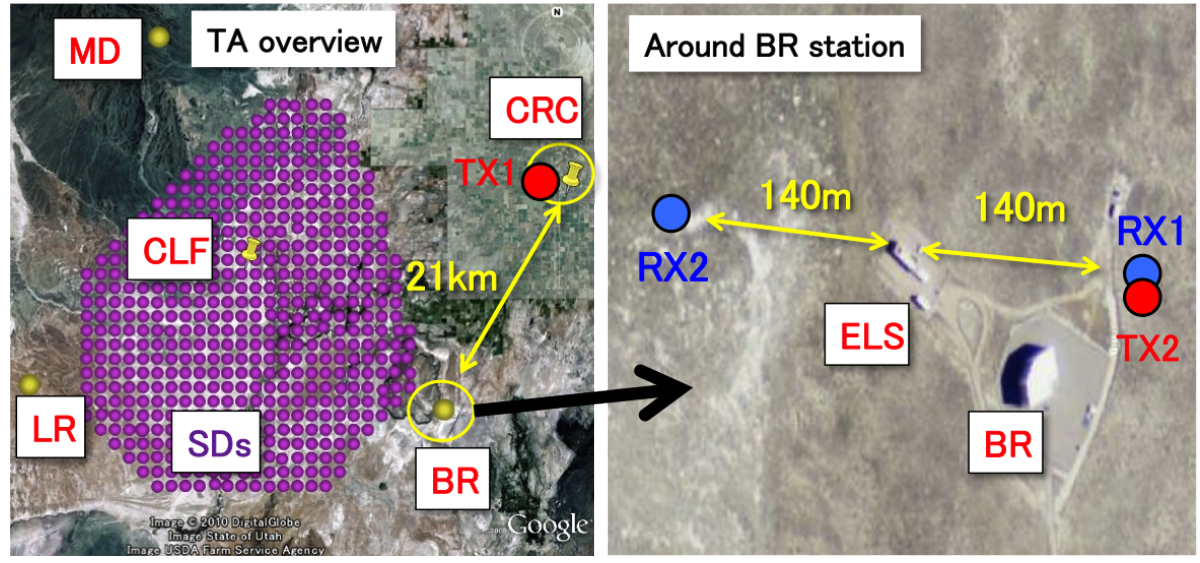

Figure 1: The overview of the positions in the electron beam test. The transmitters (TX1,TX2), receivers (RX) and ELS are shown.

$25 \mathrm{MHz}$ sampling rate and $54.1 \mathrm{MHz}$ local oscillator. The final acceptable frequency range is from $50 \mathrm{MHz}$ to $77 \mathrm{MHz}$. Those receivers store the data when the ELS trigger pulse is received. Details of this DAQ system is described in [7].

\section{Results of the electron beam test}

We have not found any signals with the $1 \mu$ s long pulse beam operation, but found a signal with the $20 \mathrm{~ns}$ short pulse beam. The example of the observed waveform is shown in Figure 2. When we shot the electron beam, a significantly strong signal appeared at the expected timing of the radar echoes. However, this signal also appeared without incident radio wave. We also measured the beam charge dependence of the observed signals and found that the observed pulse height is proportional to the output beam charge (Figure 2). From those results, the observed signal is not a radar echo signal, but a radio emission from the electron beam itself.

We also checked the polarization and frequency dependence of this signal. The polarization of the receiver can be changed by rotating the antennas. The result is that the pulse height of vertical polarization is two times larger than that of horizontal polarization. The acceptable frequency range is determined by the band pass filters and the frequency of the local oscillator. We used three band pass filters and the ratios of the pulse height from the $54.1 \mathrm{MHz}$ are as follows: 0.94 for $60 \mathrm{MHz}$, 0.02 for $175 \mathrm{MHz}$ and 0.01 for $250 \mathrm{MHz}$. The pulse height was significantly reduced when the frequency become large.

One of the theories to describe the observed radio emission is an electric field generated by the sudden appearing of the electron beam from the ELS container (sudden birth). By the calibration for the time variation of the static electric field generated by electron beam with the detector response, the expected pulse shape is in good agreement with the observed signal. It is suggested that the sudden vanishing of the secondary particles at the ground causes the radio emission (sudden death) [5]. Since these two phenomena are caused by the similar process, the observed radio emission will have the capability to use for the air shower detection. 

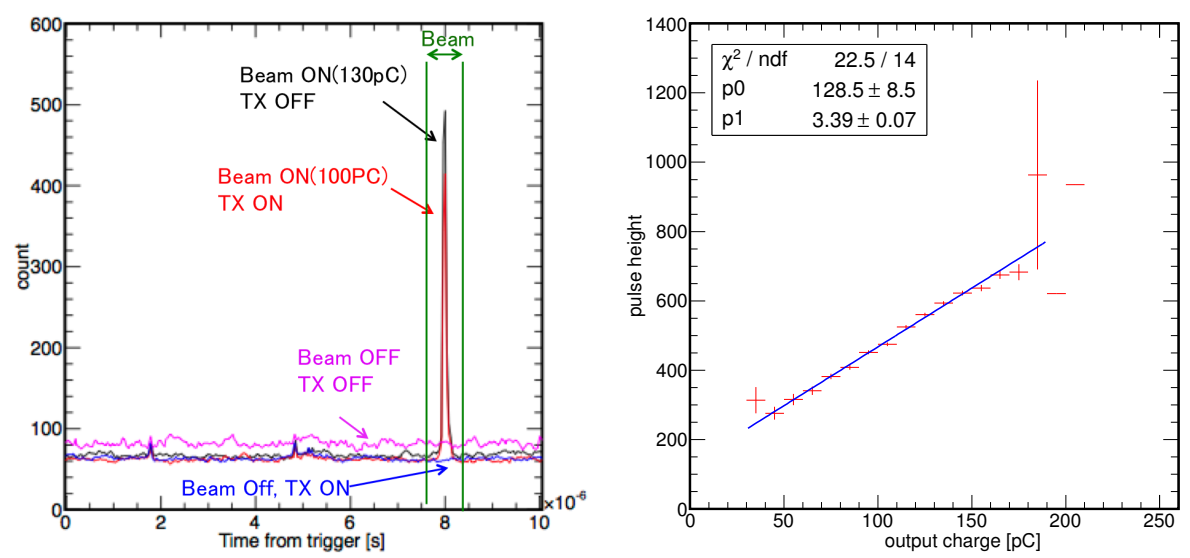

Figure 2: The signals observed in the electron beam test. The left figure shows the waveform in the four types of the experiment, the combination of beam ON/OFF and transmitter ON/OFF. The right figure is the beam charge dependence of the observed pulse height.

\section{New detector for the air shower test in the Telescope Array site}

For the confirmation of the radar echo and sudden birth signals, we developed new receivers in the TA site. The positions of the receivers are shown in Figure 3. In the TA site, there is the TARA experiment searching for the radar echoes from the UHECRs [6]. They emits the continuous radio wave of $54.1 \mathrm{MHz}$ from the transmitter which is located at the east side of the TA SD array. To use this emitted radio wave, we developed two receivers in the Central Laser Facility (CLF) at the center of the TA site. One receiver faces to the transmitter to see the front scattering of the radar echoes. This receiver expects the strongest signal for the near distance from the transmitter, but the direct observation of the transmitting wave is also strong. The other receiver faces to the opposite direction for the back scattering echoes. This expects high $\mathrm{S} / \mathrm{N}$ ratio and coincidence with TARA receivers. Because the frequency of the radar echo signal from the air showers is expected to be significantly shifted as a chirp, the wide-band receiver is required [8]. In addition, those receivers have a sensitivity for the radio emission from the air showers, geo-synchrotron and sudden death. Those receivers are triggered by the TA detectors so that we can study the sensitivities of each radio signal with the information of the air shower obtained from the TA.

The schematic view of the DAQ system is shown in Figure 4. The cross-mounted log-periodic antenna of 3-meter length, 50-500 MHz bandwidth has been installed at 6-meter height from the ground. The signal from the antenna is transferred through 30 meters of a LMR-400 coaxial cable into the DAQ system which is located inside the CLF container. The DAQ system consists of a band pass filter, 20dB pre-amplifier and the SDR (Ettus; USRP) of $25 \mathrm{MHz}$ sampling rate with the local oscillator of $54.1 \mathrm{MHz}$ frequency. The digitized signal from USRP is continuously sent to the PC via gigabit ethernet connection and stored on the RAM temporarily. The timestamps of those data are compared with the trigger time of the TA SD and matched data are stored. Because one antenna has two polarizations, four identical systems are used. Those four USRPs are synchronized with 1 PPS and $10 \mathrm{MHz}$ clocks from the GPS receiver. This GPS receiver provides the timestamps 


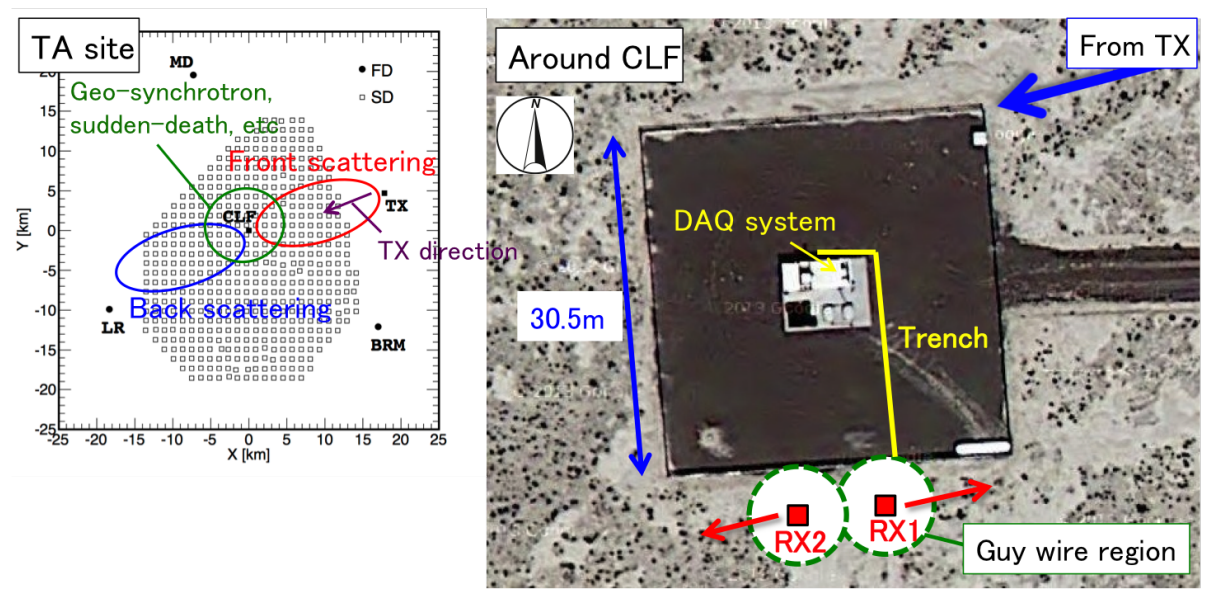

Figure 3: The left figure shows the positions of the receivers in the TA site. The right figure shows enlarged view of the positions of the receivers in the CLF site.

with $50 \mathrm{~ns}$ accuracy. This time accuracy is important to compare the observed signal to the air shower reconstructed by the TA detectors.

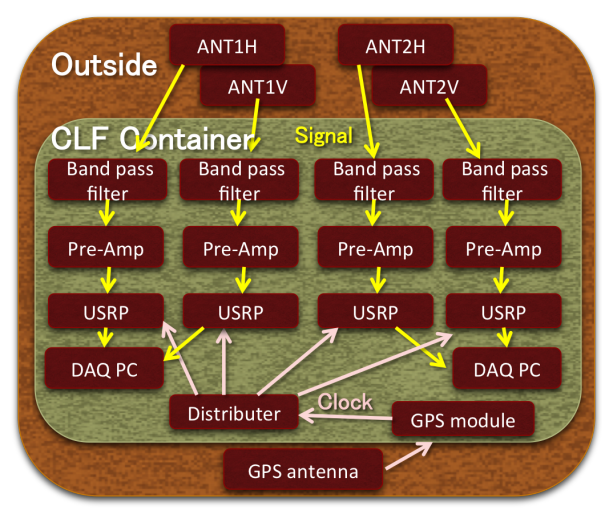

Figure 4: The schematic view of the DAQ system.

The construction of those receivers was finished in July 2014 and the steady observation was started from the October 2014. Because the polarization of the transmitter was changed from horizontal to vertical since November 2014, we recorded three types of the data, with the horizontal transmitter, no transmitting and vertical transmitter. Now the data with the vertical transmitter is recorded continuously.

\section{Conclusion}

For the study of the future huge UHECR observatory, we carried out the R\&D project to measure the air shower by the radio detection system. First the experiment with the electron beam was performed. We did not find any radar echo signal but found the radio emission signal proportional to the beam current. One of the theories to describe the observed signal is an electric field 
generated by the sudden appearing of the electron beam from the ELS container. Because the air shower is expected to emit the radio waves with the same phenomenon, this radio emission has the capability to use for the air shower detection. Second we developed the new experiment to see the radar echoes and radio emissions from the air shower. Two installed receivers which recorded the data synchronized with the TA SD are constructed at the center of the TA SD array and started the steady operation from October 2014.

\section{Acknowledgements}

The Telescope Array experiment is supported by the Japan Society for the Promotion of Science through Grants-inAid for Scientific Research on Specially Promoted Research (21000002) "Extreme Phenomena in the Universe Explored by Highest Energy Cosmic Rays" and for Scientific Research (19104006), and the Inter-University Research Program of the Institute for Cosmic Ray Research; by the U.S. National Science Foundation awards PHY-0307098, PHY- 0601915, PHY-0649681, PHY-0703893, PHY-0758342, PHY-0848320, PHY-1069280, PHY-1069286, PHY1404495 and PHY-1404502; by the National Research Foundation of Korea (2007-0093860, R3210130, 2012R1A1A2008381, 2013004883); by the Russian Academy of Sciences, RFBR grants 11-02-01528a and 13-02-01311a (INR), IISN project No. 4.4502.13, and Belgian Science Policy under IUAP VII/37 (ULB). The foundations of Dr. Ezekiel R. and Edna Wattis Dumke, Willard L. Eccles, and George S. and Dolores DorÂt'e Eccles all helped with generous donations. The State of Utah supported the project through its Economic Development Board, and the University of Utah through the Office of the Vice President for Research. The experimental site became available through the cooperation of the Utah School and Institutional Trust Lands Administration (SITLA), U.S. Bureau of Land Management, and the U.S. Air Force. We also wish to thank the people and the officials of Millard County, Utah for their steadfast and warm support. We gratefully acknowledge the contributions from the technical staffs of our home institutions. An allocation of computer time from the Center for High Performance Computing at the University of Utah is gratefully acknowledged. This work was supported by the Japan Society for the Promotion of Science through the Grand-in-Aid for the Exploratory Research Grant Number 23654078 and the Grant-in-Aid for Scientific Research (C) Grant Number 25400296.

\section{References}

[1] H. Kawai et al. , J. Phys. Soc. Jpn. Suppl. A 78 (2009) 108-113.

[2] J. Abraham et al. , Nucl. Instrum. Meth. A 523 (2004) 50.

[3] P.W. Gorham, Astropart. Phys. 15 (2001) 177-202.

[4] B.K. Shin et al. , in these proceedings.

[5] V. Marin and B. Revenu, arXiv:1211.3305 [astro-ph.HE]

[6] R. Abbasi et al. , Nucl. Instrum. Meth. A 767 (2014) 322-338.

[7] D. Ikeda et al., proceedings of 33rd International Cosmic Ray Conference, Rio, Brazil.

[8] D. Underwood, Proceedings of the 2008 IEEE Radar Conference, Rome, Italy (2008). 